\title{
ENERGY SPECTRA OF COSMIC GAMMA-RAY BURSTS
}

\author{
T. L. CLINE \\ U. D. DESAI \\ R. W. KLEBESADEL \\ I. B. STRONG
}

(NASA-TM-X-70427) ENERGY SPECTRA OF COSMIC GAMMA-RAY BORSTS (NASA) $14 \mathrm{p} \mathrm{HC}$ $\$ 3.00$

JULY 1973 


\section{ENERGY SPECTRA OF COSMIC GAMMA-RAY BURSTS}

T. L. Cline and U. D. Desai NASA/Goddard Space Flight Center Greenbelt, Maryland 20771

$$
\text { and }
$$

R. W. Klebesadel and I. B. Strong

University of California, Los Alamos Scientific Laboratory

Los Alamos, New Mexico 87544

To be published in Astrophysical Journal Letters October 1, 1973 


\section{ENERGY SPECTRA OF COSMIC GAMMA-RAY BURSTS}

T. L. Cline and U. D. Desai

NASA/Goddard Space Flight Center

Greenbelt, Maryland 20771

and

R. W. Klebesadel and I. B. Strong

University of California, Los Alamos Scientific Laboratory

Los Alamos, New Mexico 87544

ABSTRACT

We report spectral measurements of six cosmic gamma-ray bursts in the energy region of 0.1 to $1.2 \mathrm{MeV}$, made using a semi-amidirectional x-ray detector on IMP-6. These measurements confin the hard $\mathrm{X}$-ray or gamma-ray nature of the bursts, as inferred from the original observations by Klebesadel et al., (1973), and show that their maximum energy release is in this several hundred kev region. Each burst consists of several 1 or 2-seoond pulses each with the characteristic spectrum of $a \approx 150-\mathrm{keV}$ exponential, followed by a softer decay. There is no evidence of line structure in this energy region, or for a marked change in the energy spectrum within a given pulse. Event size spectra are estimated for galactic and extragalactic models; the total emission is consistent with present measurements of the diffuse background, and unlikely to account for any spectral feature in the few-MeV region.

\section{INTRODUCTION}

The occurrence of intense, several-second duration bursts of 0.1 to 1.2-MeV cosmic gamma rays, recently found using multiple Vela satellites (Klebesadel et al., 1973), has been confirmed with measurements from the IMP-6 satellite. Observations regarding times of occurrence, photon flux, 
and temporal and spectral characteristics of the bursts are entirely consistent. In particular, since the IMP-6 instrument incorporates a hard $\mathrm{X}$-ray detector with active particle rejection and full-time amnidirectional particle intensity monitoring, the results fully confirm and establish the hard $\mathrm{X}$-ray or gamma-ray nature of the incident flux.

Detailed differential energy spectra were obtained with the IMP-6 for six of the eight known events occurring during the March 1971 to september 1972 lifetime of the instrument. All of these are multiplepulse events, with several seconds separation between distinct pulses of ane or two seconds duration. The pulse spectra do not obey singleindex power laws in energy, but can be simply represented by exponentials in photon flux throughout the 100 to $1200 \mathrm{keV}$ region. The characteristic energies at maximum intensity appear to cluster near $150 \mathrm{keV}$, with indications that departures from this value can be interpreted as circumstantial, due to attenuation when the source is at great angles fram the detector axis. These burst pulses appear to ride on a softer component that exhibits a longer decay time constant, and has a characteristic exponent near $75 \mathrm{keV}$. There is no evidence for monoenergetic line structure in the several hundred kev region, or for marked changes in the spectrum with time during a single pulse. Size spectra can be estimated to predict the frequences of occurrence of smaller events for both a galactic model, e.g., a new class of gamma-ray flare star, and an extragalactic model, e.g., supernovae. In either case the total emission is 
below the value currently obtained for the diffuse celestial X-ray background, and unlikely to account for any of its spectral features.

\section{INSTRUMENTATION}

The IMP-6 satellite was launched on 14 March 71 into an elliptic orbit with an initial apogee of over $200,000 \mathrm{~km}$. Gamma-ray monitoring was provided on a nearly continuous basis, except for passes every 4.14 days through the magnetosphere, lasting several hours each. The detector was in operation from launch until 2 May 1971, and again for the period from 9 June 71 to 27 September 72. The instrument used consisted of a 2.25 -inch cliameter by 1.5-inch thick CsI(Tl) crystal, entirely surrounded by a thin plastic scintillator for particle rejection, viewed by a single PM tube. In addition to full-time monitoring of the rates of total intensity, particle intensity and gamma ray intensity, energy spectra of incident gamma rays were measured by a 14channel analyzer with simultaneous storage in all channels. The spectra were accumulated for one half of the time, for each $\approx 6.3$ second period from sun rise to sunset on the detector, determined by the optical aspect. This 50-percent duty cycle resulted in missing several of the very brief gamma-ray bursts. The spectral accumulation times were fixed at $\approx 5.1$ seconds so that the $\approx 6.3$-second life times were asynchronously split into 2 or 3 intervals of shorter durations, making possible more than one spectral determination during some of the pulses. The gain of the system was cycled through 4 positions with changes at $\approx$ one-week intervals for purposes of in-flight calibration, so that some of the bursts happened to be abserved with a 69 to $1150-\mathrm{keV}$ dymamic range and some with a 53 to 
880-keV range. The primary purpose of this gamma-ray detector was use as a coincident annihilation spectrometer incorporated in a positron detector. The secondary objective was that of a solar flare monitor, and it was in this mode of operation that these unexpected gamma ray bursts were observed.

\section{DATA OBSERVATION AND ANALYSIS}

The times of occurrence of gamma ray bursts observed with multiple Vela satellite coincidences were used to identify coincident increases in the IMP-6 gamma-ray intensity. Six of the eight Vela events were observed well above the amnidirectional background, the others being missed because of the 50-percent detector duty. cycle. It is possible that other events, of intensity too low to exceed the Vela threshold triggers, may also be observable with the IMP-6 instrument. Figure I shows the response of the IMP garma-ray detector to the event of 30 June 1971 . During a several second interval, the counts in the plastic scintillator (P) surrounding the gamma ray crystal increased by about 50, while the neutral counts in the crystal $(\gamma \overline{\mathrm{P}})$ simultaneously increased by about 18,000 . Pulses satisfying the gamma-ray logic are fed to a multichannel analyzer, fram which the outputs of three channels, added to provide the flux of 140 to $475 \mathrm{keV}$ photons, indicate an increase during one $\approx$ 5-second interval of nearly 5000 counts from a total amidirectional and secondary background of about 400 counts. This illustrates the remarkable intensity of the bursts, and shows that the response is entirely consistent with that of hard $\mathrm{x}$-rays or gamma rays.

The times of occurnence and various properties of all Vela-IMP events 


\section{PRECEDING PAGESBLANK NOT FILMED}

(Wheaton et al., 1973), contain too much emission in the X-ray region to fit $\approx 150 \mathrm{keV}$ black body spectra, and yet too little emission in the lower energies to be compared to the typical, steep X-ray spectra, having index of $\approx-3$ or more, of most hard solar flares and many celestial X-ray sources. For those pulses which were observed with sufficient temporal resolution to obtain more than one spectrum per pulse, there is no evidence for changes in the characteristic energy during its extent (not illustrated). Further, there is no evidence for line structure in this energy region. It is possible, however, that great improvements in energy and time resolution might show fine-scale spectral variability with a variety of monochromatic lines, which average out over 2 -second summations.

An integral size spectrum can be constructed, assuming a power law with index -1.5 , normalized to 6 or 8 events per 1.5 years with sizes greater than $10^{-4} \mathrm{erg} \mathrm{cm}^{-2}$ for the energy region above $100 \mathrm{keV}$. Since the 18 known events have source directions compatible with isotropy (Strong and Klebesadel, 1973) rather than with, e.g., galactic plane clustering, the source objects must either have distances in the tens to hundreds of pc if galactic, or, if extragalactic in nature, have distances of > several Mpc. Thus, this size spectrum can be normalized for these two . models in order to obtain predictions of the frequencies of occurrence of smaller events. In the case of extragalactic sources, e.g., ganma-ray r_ch and optically poor supemovae or other large collapsing objects, a sumation of all emissions up to cosmological distances produces a total isotropic background intensity which is below the presently observed 
diffuse cosmic background in this energy interval. Thus an extragalactic origin cannot be ruled out. Further, if all sources have spectra with $\approx 150-\mathrm{keV}$ exponentials, then the total cosmic spectrum will not extend into the several MeV region with sufficient intensity to explain the bump in the diffuse cosmic background observed (Trombka et al., 1973) at those energies.

\section{References}

Klebesadel, R. W., Strong, I. B., and Olson, R. A., 1973, Ap. J. Letters 182, L85.

Strong, I. B., and Klebesadel, R. W., 1973 (in preparation).

Trombka, J. I., Metzger, A. E., Arnold, J. R., Matteson, J. L., Reedy, R. C., and Peterson, L. E., 1973, Ap. J. 181, 737.

Wheaton, W. A., Ulmer, M. P., Baity, W. A., Datlowe, D. W., Elcan, M. E., Peterson, L. E., Klebesadel, R. W., Strong, I. B., Cline, T. L., and Desai, U. D., 1973 (submitted for publication). 


\begin{tabular}{|c|c|c|c|c|}
\hline EVENT & BURST & $I_{Q}$ & $\underline{E_{0}}$ & IOOK ANGTE \\
\hline $15 \operatorname{Mar} 71$ & Second Max & 1.9 & 156 & Includes Source $\left(\alpha \approx 50^{\circ}, \delta=-30 \pm 10^{\circ}\right)$ \\
\hline $18 \operatorname{Mar} 71$ & Decay of lst & 1.8 & 74 & Spins through source \\
\hline $30 \mathrm{Jun} 71$ & First Max & 0.7 & 276 & Source below satellite horizon \\
\hline $30 \mathrm{Jun} 71$ & Second Max & 5.5 & 142 & Spins through Source \\
\hline $30 \operatorname{Jun} 71$ & Decay of 2 nd & 0.7 & 110 & Spins through source \\
\hline $17 \mathrm{Jan} 72$ & Decay of 1 st & 0.10 & 138 & Source position undetermined \\
\hline $17 \operatorname{Jan} 72$ & Second Max & 0.35 & 184 & Source position undetemined \\
\hline $17 \operatorname{San} 72$ & Decay & 0.11 & 170 & Source position undetermined \\
\hline $28 \operatorname{Mar} 72$ & First Max & 0.50 & 238 & Source near or below horizon \\
\hline $28 \operatorname{Mar} 72$ & Second Max & 0.55 & 176 & Source position undetemined \\
\hline 14 May 72 & First Max & 0.8 & 166 & Inclucles Source $\left(\alpha \approx 175^{\circ}, \delta \approx+77^{\circ}\right)$ \\
\hline 14 May 72 & Second Max & 0.8 & 152 & Includes Source \\
\hline
\end{tabular}

Table 1. Characteristics of gamma-ray burst spectra.

Exponential fits in $\mathrm{d} / \mathrm{dE}$ provide $\mathrm{I}_{\mathrm{O}}$ in units of photons $\mathrm{am}^{-2} \mathrm{keV}^{-1}$, and $E_{O}$ in units of keV, both of which have systematic uncertainties depending on relative look angle. 


\section{Figure Captions}

Figure 1. The response of the detector to a gamma ray burst, as indicated by the plastic anticoincidence (P), the CsI gamma ray detector $(\gamma \overline{\mathrm{P}})$ and several channels added to give the 140 to $475 \mathrm{kev}$ photon rate, where the gamma-ray energy response is maximized. Each point samples two differential energy spectra.

Figure 2a. Number spectra dn/dE, of several bursts, selected for the greatest variety of responses. The harder spectra are interpreted as due to attenuation of the incident beam by the satellite material in cases where the source was below the detector horizon. Fig. 2b (insert). The energy spectrum, $E \mathrm{dn} / \mathrm{dE}$, of a directly observed event. 


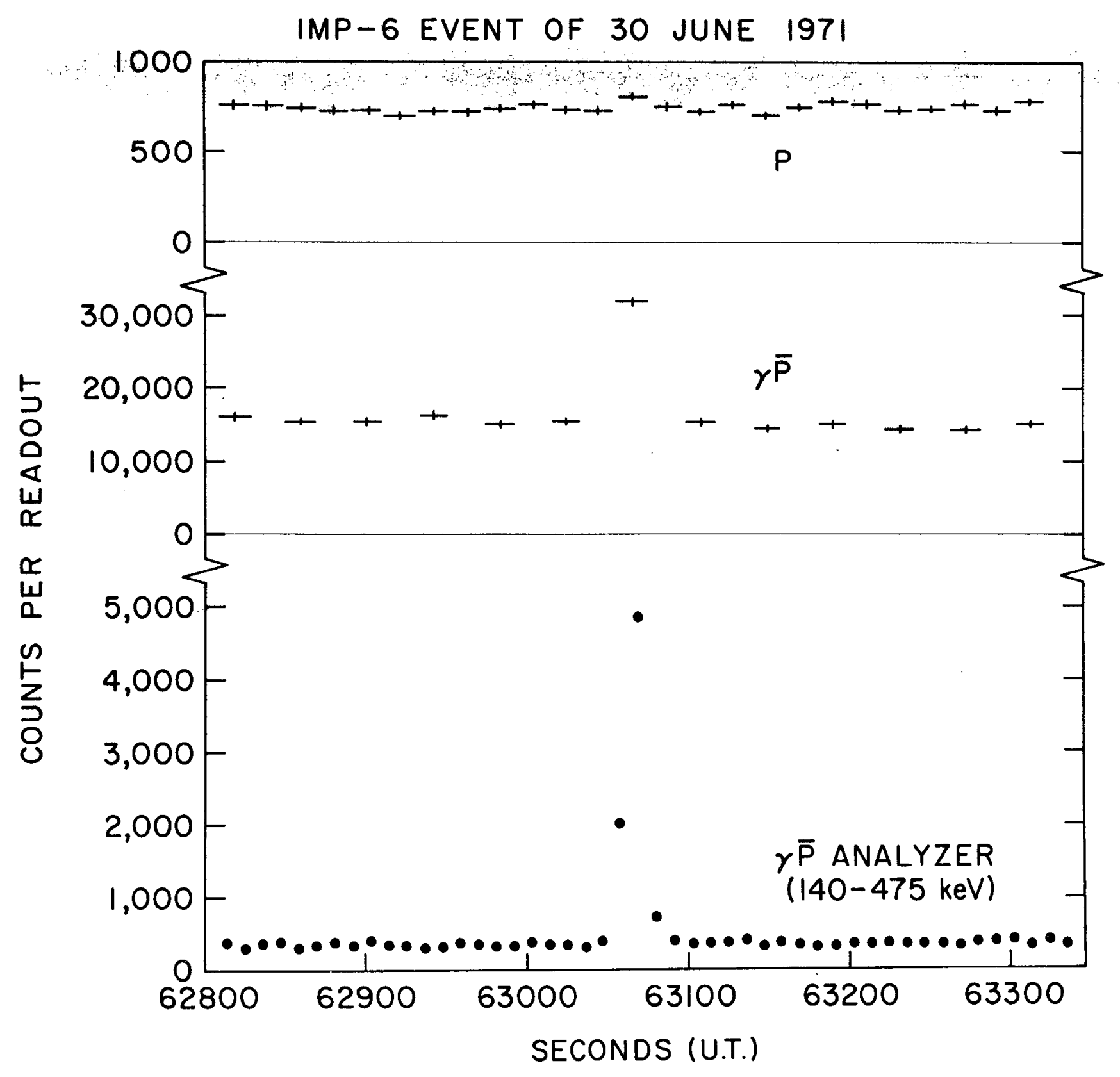




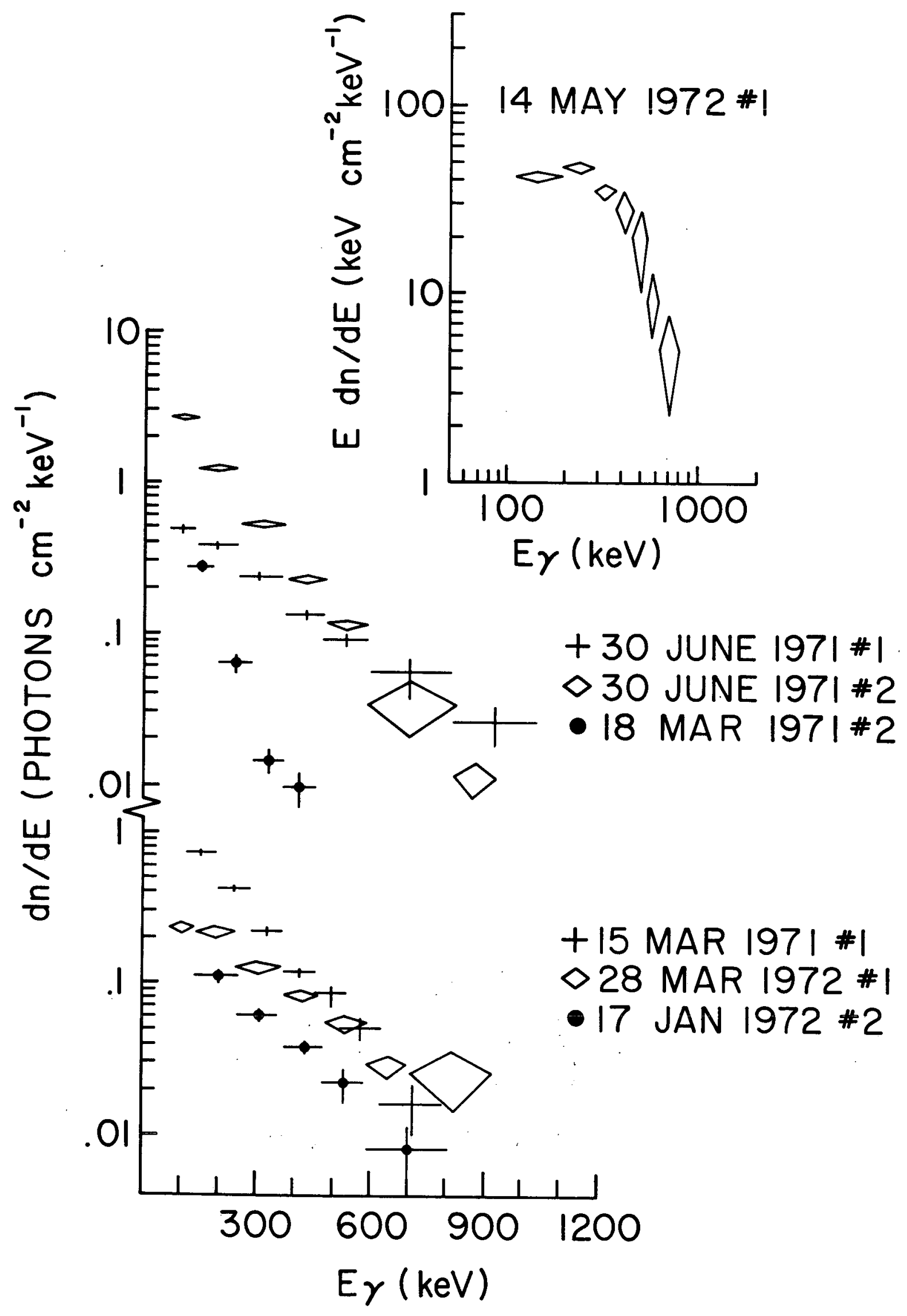

